\title{
New nearby stars selected in a high proper motion survey by DENIS photometry
}

\author{
C. Reylé ${ }^{1}$, A. C. Robin ${ }^{1}$, R.-D. Scholz ${ }^{2}$, and M. Irwin ${ }^{3}$ \\ 1 CNRS UMR 6091, Observatoire de Besançon, BP 1615, 25010 Besançon Cedex, France \\ 2 Astrophysikalisches Institut Potsdam, An der Sternwarte 16, Potsdam, 14482, Germany \\ 3 Institute of Astronomy, University of Cambridge, Madingley Road, Cambridge, CB3 OHA, UK \\ e-mail: celine@obs-besancon.fr; annie.robin@obs-besancon.fr; rdscholz@aip.de; mike@ast.cam.ac.uk
}

Received 22 February 2002 / Accepted 30 April 2002

\begin{abstract}
We present new nearby stars extracted from a proper motion catalogue and having a DENIS counterpart. Their distances and spectral type are estimated using the DENIS colours. 107 stars are within 50 pc. 31 stars among them have previously measured distances. In addition, 40 stars may enter within the $50 \mathrm{pc}$ limit depending on which population they belong to. 6 stars among them have already measured distances. 5 objects, LHS5045, L225-57, LP831-45, LHS1767, and WT792, are probably closer than 15 pc, with L225-57 at 9.5 pc. Most of these stars are M-type while 4 stars are white dwarfs. $88 \mathrm{M}$-dwarfs are disc stars, 14 belong to the thick disc and 1 to the spheroid.
\end{abstract}

Key words. Galaxy: solar neighbourhood - stars: late-type - stars: white dwarfs

\section{Introduction}

The solar neighbourhood serves as a fundamental constraint for our understanding of the stellar physics and the Galaxy. The nearest stars provide accurate data on luminosities, temperatures, masses, which are fundamental parameters of stellar astronomy. Their kinematics, chemical composition, and mass function also hold important clues to the nature and history of the Milky Way.

However, among the nearest stars, many of the low luminosity stars such as white, red, and brown dwarfs remain undetected. Henry et al. (1997) estimated that more than 30\% of the stars within $10 \mathrm{pc}$ are currently missing from the solar neighbourhood sample. The deficit is estimated to be twice within 25 pc (Henry et al. 2002) and is largest south of decination $-30^{\circ}$. As a confirmation, stars are continuously identified as nearby stars in proper motion catalogues or in Schmidt plates (Henry et al. 1997; Henry et al. 2002; Gizis \& Reid 1997; Scholz et al. 1999; Scholz et al. 2001; Scholz et al. 2002; Jahreiss et al. 2001; Phan-Bao et al. 2001; Delfosse et al. 2001).

In addition, new nearby faint stars, mainly brown dwarfs, are detected in deep sky surveys (Ruiz et al. 1997; Delfosse et al. 1997; Delfosse et al. 1999; Martín et al. 1999; Kirkpatrick et al. 1999; Kirkpatrick et al. 2000; Reid et al. 2000; Fan et al. 2000; Gizis et al. 2000).

One way to detect nearby stars is to select the red faint objects obtained in near-infrared surveys: the Two Micron All

Send offprint requests to: C. Reylé, e-mail: celine@obs-besancon.fr
Sky Survey (2MASS) (Kirkpatrick et al. 1999; Kirkpatrick et al. 2000; Reid et al. 2000; Gizis et al. 2000) and the Deep NearInfrared Survey of the Southern Sky (DENIS) (Delfosse et al. 2001; Phan-Bao et al. 2001). Most of the nearest stars found in these surveys turned out to be high proper motion objects. Given this fact, Scholz et al. (2001) searched for new stars in the solar neighbourhood by combining proper motion catalogues with the 2MASS data base.

Using the same approach, we cross-identify a proper motion catalogue with DENIS data and estimate the distances using near-infrared photometry. The proper motion catalogue is described in Sect. 2. In Sect. 3, we give the result of the crossidentification with the DENIS data base. Section 4 presents the distance estimation. In Sect. 5, we discuss the effect of metallicity on the distance estimation. In Sect. 6, we list the stars found to be within $50 \mathrm{pc}$ and discuss the precision on our distance determinations.

\section{High proper motion sample}

One of the criteria for finding nearby stars is their large proper motion. The main source for the identification of such stars are the high-proper motion catalogues of Luyten: the Luyten Half Second proper motion catalogue (LHS) and the New Luyten Catalogue of Stars with Proper Motions Larger than Two Tenths of an Arcsecond (NLTT) based on observations with the Palomar Schmidt telescope. Dawson (1986) showed that the LHS catalogue is incomplete south of declination $-33^{\circ}$, 
$R>18$ or $\mu>2.5^{\prime \prime} \mathrm{yr}^{-1}$. Wroblewski \& Torres $(1989,1991$, 1994, 1996, 1997) improved the completness in the southern sky and found new stars with $\mu>0.15^{\prime \prime} \mathrm{yr}^{-1}$ and photographic magnitudes $m_{\mathrm{pg}}<20$. Ruiz et al. (1993) found proper motion stars in the ESO Schmidt plates with $\mu>0.1^{\prime \prime} \mathrm{yr}^{-1}$ and $R<20$.

In order to complete the existing proper motion catalogues at fainter magnitudes in the southern hemisphere, Scholz et al. (2000) used Automatic Plate Measuring (APM) measurements of sky survey plates taken with the UK Schmidt telescope. Plates in the passbands $B_{\mathrm{J}}$ and $R$ were taken with typical epoch difference of 15 years. The resulting catalogue contains 693 stars (out of which 195 have already been published by Scholz et al. 2000) south of declination $-15^{\circ}$, in the regions $0^{\mathrm{h}}-7^{\mathrm{h}}$, $10^{\mathrm{h}}-14^{\mathrm{h}}$, and $19^{\mathrm{h}}-23^{\mathrm{h}}$ in right ascension. The overall search area is about 3000 square degrees. The lower proper motion limit of the survey is typically $0.25 \operatorname{arcsec} \mathrm{yr}^{-1}$. But in fields with smaller epoch difference between the $B_{\mathrm{J}}$ and $R$ plates, only objects with even larger proper motions could be detected, respectively. The distribution of the epoch differences as a function of declination was shown in Scholz et al. (2000). The largest proper motions found are about $1.3 \operatorname{arcsec} \mathrm{yr}^{-1}$. The practical limit for searching for high proper motion objects using two plates in different passbands is about 1 magnitude above the respective plate limits, i.e. $R=20.0$ and $B_{\mathrm{J}}=21.5$. For a comparison with other high proper motion catalogues and an estimate of the completeness of the survey, see Scholz et al. (2000). About half of the objects detected are known proper motion stars, whereas the remaining objects mainly at fainter magnitudes are new high proper motion stars.

With these measurements, they were able to fill the gap in the high proper motion survey of faint stars with $\delta<-33^{\circ}$. In addition they obtained more accurate positions and photometry for the already known high proper motion stars. The accuracies are 0.1 to $0.2^{\prime \prime}$ in position, $0.03^{\prime \prime} \mathrm{yr}^{-1}$ on proper motion, and $\sim 0.2$ mag on photometry.

\section{Cross-identification with DENIS}

The DENIS survey (Epchtein et al. 1997) provides a full coverage of the southern sky in the optical band $I(0.85 \mu \mathrm{m})$ and the near-infrared bands $J(1.25 \mu \mathrm{m})$ and $K_{\mathrm{s}}(2.17 \mu \mathrm{m})$. The position accuracy is $0.5^{\prime \prime}$ and the photometric accuracy is better than $0.1 \mathrm{mag}$. About $40 \%$ of the data are up to now calibrated at the Paris Data Analysis Center (PDAC).

We look for counterparts of the given proper motion sample in the DENIS point source catalogue extracted at the PDAC with a search radius of $3^{\prime \prime}$. We get 301 recoveries among the 693 objects. None of them have a counterpart in the USNO A2.0 catalogue, as expected for proper motion stars. The density of stars with no USNO counterpart in a field in the Galactic plane is less than 0.01 star within the search region. Thus the possibility of false cross-identification is low, smaller than $1 \%$. Most of the unrecovered stars are in the unprocessed part of the DENIS survey. Dots in Fig. 1 shows the $(I, I-J)$ colourmagnitude diagram of the recovered stars. Most stars have $I-J>1$. They are M-dwarfs (Leggett 1992). Stars with $0.5<I-J<1$ are G or K-dwarfs. 1 star, with $I-J>3$, is a possible brown dwarf (square). Stars with $I-J<0.5$ are

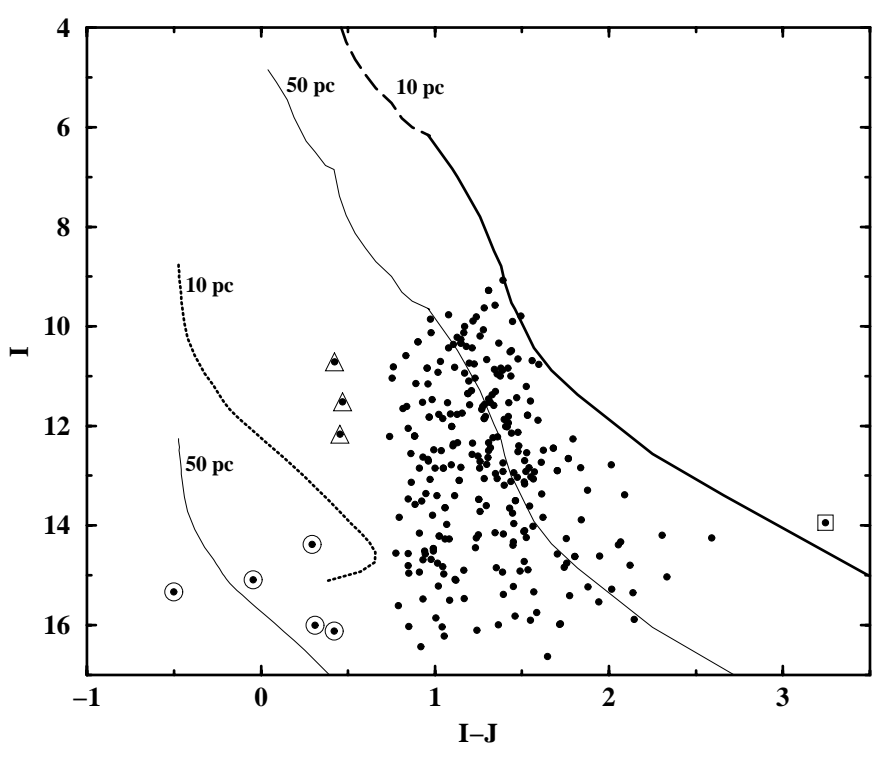

Fig. 1. $(I, I-J)$ colour-magnitude diagram for the high proper motion stars cross-identified with DENIS. Dots: G, K, or M-dwarfs. Square: possible brown dwarf. Circles: white dwarfs. Triangles: G-subdwarfs. Solid line: theoretical relation for M-dwarfs with solar metallicity at 10 pc (Baraffe et al. 1998). Dashed line: theoretical relation for $G$ and K-dwarfs with solar metallicity at $10 \mathrm{pc}$ (Lejeune et al. 1997). Dotted line: theoretical relation for white dwarfs with $0.6 M_{\odot}$ at $10 \mathrm{pc}$ (Bergeron et al. 1995). The thin lines show the same relations for stars at $50 \mathrm{pc}$.

either white dwarfs (circles) or distant subdwarfs (triangles), as discussed in Sect. 4.

\section{Photometric distance estimation}

Photometric distances are estimated using the $I-J$ colour index. Theoretical colour-magnitude relations $\left(M_{I}, I-J\right)$ for stars placed at $10 \mathrm{pc}$ are superimposed to the observations in Fig. 1. The solid line shows the relation for M-dwarfs with solar metallicity (Baraffe et al. 1998), the dashed line shows the relation for solar metallicity G and K-dwarfs (Lejeune et al. 1997), and the dotted line shows the relation for white dwarfs with $0.6 M_{\odot}$ (Bergeron et al. 1995).

We compute photometric distances from the DENIS I $J$ colour index and the corresponding theoretical colourmagnitude relation: Baraffe et al. (1998) relation for M-dwarfs with $I-J>1$, Lejeune et al. (1997) relation for G and K-dwarfs in the range $0.5<I-J<1$. Stars with $I-J<0.5$ can be either nearby white dwarfs or distant subdwarfs. The 5 faintest blue stars (circles in Fig. 1) are white dwarfs. Indeed if they would be subdwarfs with that faint apparent magnitude, they would be at $d>1600 \mathrm{pc}$. Their proper motion would lead to a tangential velocity $>4000 \mathrm{~km} \mathrm{~s}^{-1}$, much larger than the Galactic escape speed (Leonard \& Tremaine 1990; Meillon et al. 1997). For these stars, we use Bergeron et al. (1995) model atmosphere for white dwarfs of mass $0.6 M_{\odot}$, before the turnover $\left(M_{I} \lesssim\right.$ 14.5), assuming they are not cool white dwarfs.

The 3 brighter blue stars (triangles in Fig. 1) are more probably late G-stars of the spheroid with large tangential velocity, rather than disc white dwarfs closer than $3 \mathrm{pc}$. The theoretical 
relation from Lejeune et al. (1997) for the typical spheroid metallicity $\left[\frac{\mathrm{Fe}}{\mathrm{H}}\right]=-1.8$ gives $M_{I} \sim 5 \mathrm{mag}$ at $I-J \sim 0.5$. The derived photometric distances of these 3 stars ranges between 160 and $260 \mathrm{pc}$, and their tangential velocities between 280 and $380 \mathrm{~km} \mathrm{~s}^{-1}$, in agreement with the spheroid kinematics.

$I-K_{\mathrm{s}}$ is also a good estimator of the luminosity or effective temperature of M-dwarfs. Photometric distances differ by $15 \%$ depending on which colour index is used. However, Delfosse et al. (1997) showed that the $K_{\mathrm{s}}$ band of DENIS can differ by $\sim 0.1$ mag compared with the standard $K$ band for which models are computed. We then choose to estimate the distances from the $I-J$ colour index and $\left(M_{I}, I-J\right)$ theoretical relations.

\section{Metallicity effect}

Within the sample magnitude range and proper motion range, one expects to get disc stars, but also thick disc and spheroid stars. Using a theoretical colour-magnitude relation at solar metallicity would overestimate the distance for the stars of these old populations. We use the Besançon model of population synthesis to reproduce the stellar content in the fields of the sample. The Besançon model has been described in Haywood et al. (1997). Since then, Robin et al. (2000) improved the halo density law and mass function, while Reylé \& Robin (2001) constrained the thick disc population description. New constraints available on the galactic potential, the local luminosity function and the kinematics versus age, obtained with the Hipparcos mission, are also taken into account to derive the thin disk density law (Robin et al., to be submitted).

The simulation shows that the different populations are separated in the plane $(H, I-J)$, where $H=I+5 \log \mu+5$ is the reduced proper motion. We define three regions in this plane where stars are most probably disc stars, thick disc stars, or spheroid stars, as represented in Fig. 2.

We determine the photometric distance using a theoretical colour-magnitude relations $\left(M_{I}, I-J\right)$ at solar metallicity for stars in the disc region, at metallicity $\left[\frac{\mathrm{Fe}}{\mathrm{H}}\right]=-0.8$ in the thick disc region, and at metallicity $\left[\frac{\mathrm{Fe}}{\mathrm{H}}\right]=-1.8$ in the spheroid region. These relations are obtained by interpolation of the available relations with $\left[\frac{\mathrm{Fe}}{\mathrm{H}}\right]=-0.5,-1,-1.5,-2$ from Lejeune et al. (1997) for G and K-stars and Baraffe et al. (1998) (private communication for low metallicities) for M-stars.

For stars in between two regions, we make two determinations with both metallicity hypotheses (disc/thick disc or thick disc/spheroid). The probability that a star with a magnitude $I$, a colour index $I-J$ and a proper motion $\mu_{\text {tot }}$, belongs to one population is estimated from the population distribution of the stars in the simulated sample. In this case, both distances and tangential velocities are computed.

Disc stars with tangential velocities as large as $250 \mathrm{~km} \mathrm{~s}^{-1}$ still are found with a large probability in the simulated sample. Indeed, the sample is not representative of the entire disc population, but as a high proper motion sample, it selects the stars having a high velocity.

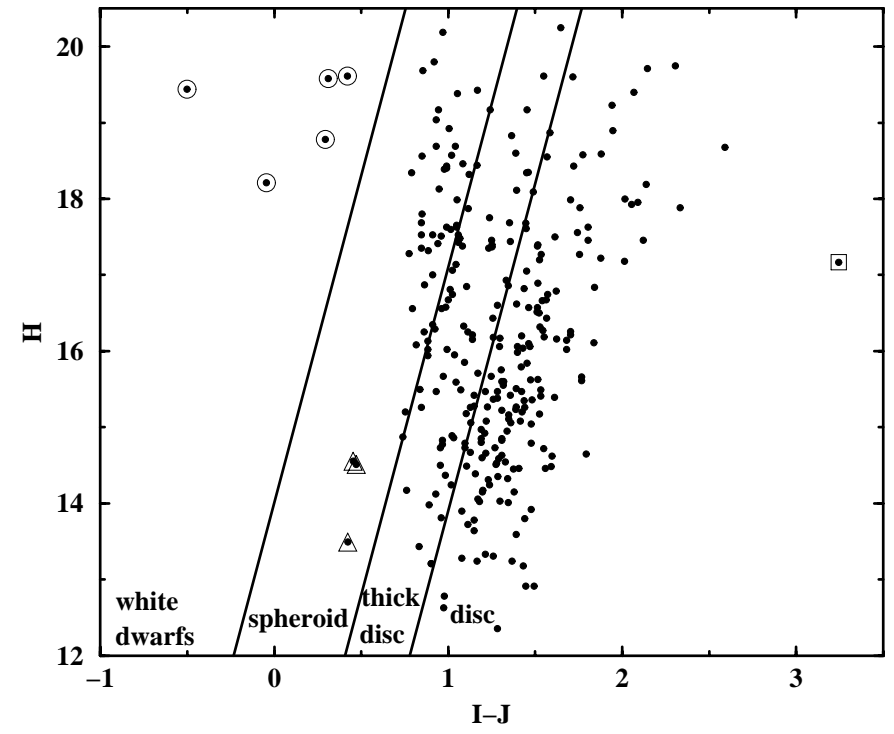

Fig. 2. $(H, I-J)$ diagram for the high proper motion stars crossidentified with DENIS. Symbols have the same meaning as in Fig. 1. The solid lines define the regions where the stars are most probably, from right to left: disc stars, thick disc stars, spheroid stars, white dwarfs.

\section{Stars within $50 \mathrm{pc}$}

107 stars are found to have photometric distances less than 50 pc. They are mainly M-stars, but 4 stars are white dwarfs. According to the colour-reduced proper motion diagram ( $H, I-$ $J), 88$ stars among them belong to the disc, 14 to the thick disc stars, and 1 to the spheroid. In addition, 33 stars belong either to the disc or to the thick disc. Distance estimations with both hypothesis are made for these stars and they are possibly within $50 \mathrm{pc}$. Likewise, 7 stars belong either to the spheroid or to the thick disc and may enter or not within the $50 \mathrm{pc}$ limit.

Astrometric, photometric and kinematical characteristics, estimated distances and spectral types are given in Table 1 . The spectral types for M-stars are derived from the $I-J$ colour index (Leggett 1992). 4 late M-type stars have been previously identified by Phan-Bao et al. (2001) and 1 by Henry et al. (2002). 6 stars already have spectroscopic distance estimates (Scholz et al. 2002). The extremely red object is the already known brown dwarf LP944-20 at 5 pc (Tinney 1996). Trigonometric distances from the Yale parallax catalogue or the Hipparcos catalogue, or photometric distances from the ARI database for nearby stars (ARICNS), available for 25 stars, are also indicated in Table 1.

Among the list of 107 nearby stars, 15 new stars are within the 25 pc limit of the Catalogue of Nearby Stars (CNS3, Gliese \& Jahreiss 1991). New discoveries within 15 pc are LHS5045, L225-57, LP831-45, LHS1767, and WT792. L225-57 is presumably closer than $10 \mathrm{pc}$, at $d=9.5 \mathrm{pc}$. Its estimated spectral type is M3.5. The star LHS124 may be a M1-star of the thick disc at $7.2 \mathrm{pc}$, although the simulation of the stellar sample tends to show it is more likely a more distant star in the disc. The distance indicated in the ARICNS $(21 \mathrm{pc})$ seems also to favor a disc star. 
Table 1. Nearby stars candidates. The distance $d$ is estimated from the $I-J$ colour index. When two distances are indicated, they are obtained with two metallicity hypothesis and their probable population with probability are indicated in the last two columns (see text): $\mathrm{I}=$ disc, int $=$ thick disc, II $=$ spheroid. The tangential velocity is also computed with both distance estimates. The spectral types for M-stars are derived from Leggett (1992). wd means white dwarf.

\begin{tabular}{|c|c|c|c|c|c|c|c|c|c|c|c|c|c|c|c|c|}
\hline $\begin{array}{l}\text { name } \\
\text { APMPM }\end{array}$ & $\begin{array}{l}\text { other } \\
\text { name }\end{array}$ & $\alpha_{\mathrm{J} 2000}$ & $\delta_{\mathrm{J} 2000}$ & $\begin{array}{l}\text { UKST } \\
\text { epoch }\end{array}$ & $\begin{array}{c}R \\
\text { UKST }\end{array}$ & $\begin{array}{l}B_{\mathrm{J}}-R \\
\text { UKST }\end{array}$ & $\begin{array}{c}I \\
\text { DENIS }\end{array}$ & $\begin{array}{c}I-J \\
\text { DENIS }\end{array}$ & $\begin{array}{l}J-K_{\mathrm{S}} \\
\text { DENIS }\end{array}$ & $\begin{array}{c}d \\
\text { (pc) }\end{array}$ & $\mu_{x}$ & ${ }_{-1}{ }^{\mu_{y}}$ & $\begin{array}{c}V_{\mathrm{t}} \\
\left(\mathrm{km} \mathrm{s}^{-1}\right)\end{array}$ & type & \multicolumn{2}{|c|}{ population \% } \\
\hline J0001-3650 & LP987-71 & 000034.09 & -365009.3 & 1996.61 & 13.28 & 1.75 & 12.78 & 1.09 & 0.82 & 43.9 & 0.40 & 0.11 & 85.3 & M0.5 & int & \\
\hline J0003-3414 ${ }^{1}$ & LHS1008 & 000239.95 & -341337.7 & 1996.61 & 14.22 & 0.45 & 14.38 & 0.2 & 0.63 & 17.5 & 0.15 & -0.74 & 63.0 & wd & & \\
\hline $\mathrm{J} 0009-2707^{2}$ & LHS1026 & 000903.88 & -270722.2 & 1990.80 & 10.08 & 1.92 & 9.76 & 1.08 & 0.64 & $40.5 / 11.6$ & 0.66 & 0.11 & $128.6 / 36.8$ & M0.5 & I/int & $85 / 15$ \\
\hline J0013-3757 & WT3 & 001329.87 & -375644.8 & 1989.66 & 13.24 & 2.29 & 12.85 & 1.0 & 0.78 & $73.8 / 33.7$ & 0.07 & -0.58 & 204.4/93.3 & M0 & int/II & $85 / 15$ \\
\hline J0015 & L218-29 & 001 & -51 & 69 & 11.04 & 2. & 11.15 & 0. & 0.84 & $100.0 / 42.6$ & 0.33 & 0.09 & $161.2 / 68.7$ & 10 & int & $35 / 65$ \\
\hline J0017-4149 & WT4 & 0016 & -414919.1 & 1989.66 & 14.80 & 2.1 & 13.98 & 1. & 0.89 & $80.5 / 44.0$ & 0.37 & -0.33 & 189.2/103.4 & M0.5 & int/II & $50 / 50$ \\
\hline $\mathrm{J} 0017-5016^{3}$ & L218-9 & 001636.18 & -501610.9 & 1993.69 & 10.12 & 2.4 & 10.12 & 1. & 0.88 & 38.1 & 0.31 & 0.29 & 75.8 & M1 & I & \\
\hline J0020-5743 & L170-67 & 002 & -5742 & 15 & 13.11 & 2. & 12.02 & & 0.80 & 35.8 & 0.39 & 0.12 & 69.6 & M3 & I & \\
\hline $\mathrm{J} 0023-4833$ & & 002234.65 & -4832 & 1993.69 & 13.72 & 2. & 12.83 & 1. & 0.97 & $138.8 / 35.2$ & 0.31 & 0.10 & 217.1/55.1 & M0.5 & I/int & $35 / 65$ \\
\hline J0033-4733 & L291-115 & 003313.26 & -473317.8 & 1993.69 & 14.20 & 2.25 & 12.26 & 1. & 0.94 & & 0.26 & 0.15 & 22.3 & M5 & & \\
\hline J0034-3759 & LP937- 57 & 003342.79 & -375910.5 & 989.66 & 11.74 & 1.84 & 11.04 & 1. & 0.74 & 48.3 & 0.18 & 0.41 & 103.0 & M1 & 1 & \\
\hline J0049-6102 ${ }^{4}$ & LHS124 & 004927.58 & -610232.4 & 1990.78 & 10.52 & 1.51 & 9.89 & 1. & 0.76 & $29.5 / 7.2$ & 1.09 & -0.04 & $52.4 / 37.2$ & M1 & I/int & $75 / 25$ \\
\hline J0058-2751 & LHS129 & 005826.55 & -275121.7 & 1986.77 & 10.28 & 1.87 & 9.28 & 1. & 1.14 & 16.4 & 1.26 & -0.32 & 101.1 & M2.5 & I & \\
\hline 059-3127 & LHS1171 & 005844.47 & 312709.0 & 1986.77 & 12.93 & 2.24 & & 1.31 & 0.75 & 44.7 & 0.50 & -0.40 & 135.6 & M2.5 & & \\
\hline J0102-6739 & LHS1178 & 10205.88 & -673920.2 & 1987.82 & 13.84 & 1.80 & 12.85 & 105 & 0.75 & $55.2 / 28.4$ & 0.88 & -0.18 & $35.0 / 120.9$ & M0 & int/II & $50 / 50$ \\
\hline J0127-3219 & LHS1251 & 12702.57 & -321850.2 & 1991.91 & 12.28 & 2. & & & 0.81 & 36.0 & 0.20 & -0.52 & 95.6 & M0 & int & \\
\hline J0149-6427 & L88-1 & 14832.97 & -642644.0 & 1987.90 & 12.02 & 1.82 & 10.92 & 1 & 0.86 & $79.3 / 26.7$ & 0.41 & & $172.9 / 58.2$ & M0 & $\mathrm{I} / \mathrm{int}$ & $30 / 70$ \\
\hline J0150-3319 & LP940-20 & 014942.37 & -331921.5 & 1991.91 & 14.36 & 2.2 & 12.65 & 1.77 & 0.88 & 19.6 & 0.38 & & 37.2 & M5 & $\mathrm{J}$ & \\
\hline J0151-3058 & $\begin{array}{l}\text { LP884-94 } \\
\text { LUS5045 }\end{array}$ & 015104.98 & -305759.0 & 1994.89 & 14.02 & 2.60 & 12.52 & 1.47 & 001 & 35.5 & 0.08 & 0.31 & 53.8 & M3.5 & I & \\
\hline J0153-4806 & LHS5045 & 015252.07 & -480539.2 & $\begin{array}{l}1988.91 \\
10909\end{array}$ & $\begin{array}{l}12.40 \\
13.65\end{array}$ & $\begin{array}{l}2.12 \\
254\end{array}$ & 10.77 & 1.60 & 0.91 & 11.0 & -0.55 & -0.20 & 30.8 & M4 & I & \\
\hline $\begin{array}{l}\text { J0155-5307 } \\
\text { J0158-5031 }\end{array}$ & L223-77 & $\begin{array}{l}015512.82 \\
015754.88\end{array}$ & $\begin{array}{l}-530634.0 \\
-503056.1\end{array}$ & $\begin{array}{l}1990.93 \\
1989.91\end{array}$ & $\begin{array}{l}13.65 \\
17.29\end{array}$ & $\begin{array}{l}2.54 \\
2.23\end{array}$ & $\begin{array}{l}12.13 \\
14.61\end{array}$ & $\begin{array}{l}1.48 \\
1.95\end{array}$ & 0.88 & $\begin{array}{l}29.6 \\
37.8\end{array}$ & $\begin{array}{l}0.26 \\
0.71\end{array}$ & $\begin{array}{r}0.22 \\
-0.10\end{array}$ & 47.7 & $\begin{array}{l}\text { M3.5 } \\
M 5.5\end{array}$ & I & \\
\hline $\begin{array}{l}\mathrm{J} 0158-5031 \\
J 021-6314^{6}\end{array}$ & LHS1351 & $\begin{array}{l}015754.88 \\
02111.04\end{array}$ & $\begin{array}{l}-503056.1 \\
-6313376\end{array}$ & $\begin{array}{l}1988.91 \\
1989.73\end{array}$ & 10.77 & $\begin{array}{l}2.23 \\
2.08\end{array}$ & $\begin{array}{r}14.61 \\
9.80\end{array}$ & $\begin{array}{l}1.95 \\
1.24\end{array}$ & 0.81 & $\begin{array}{l}37.8 \\
26.8\end{array}$ & $\begin{array}{r}0.71 \\
-0.70\end{array}$ & $\begin{array}{l}-0.10 \\
-0.31\end{array}$ & 129.0 & M5.5 & I & \\
\hline $\begin{array}{l}\mathrm{J} 0211-6314^{6} \\
\text { J0211-4714 }\end{array}$ & & $\begin{array}{l}021119.04 \\
02112670\end{array}$ & $\begin{array}{l}-631337.6 \\
-4713403\end{array}$ & & 13.75 & $\begin{array}{l}2.0 \\
1.8\end{array}$ & 13. & 1. & $\begin{array}{l}0.81 \\
0.80\end{array}$ & $\begin{array}{l}26.8 \\
413\end{array}$ & $\begin{array}{r}-0.70 \\
0.35\end{array}$ & $\begin{array}{r}-0.31 \\
0.23\end{array}$ & $\begin{array}{l}97.8 \\
82 .\end{array}$ & M1 & I & \\
\hline $\begin{array}{l}\mathrm{J} 0211-4714 \\
\mathrm{~J} 0213-3352\end{array}$ & LHS1355 & $\begin{array}{l}021126.70 \\
021239.48\end{array}$ & $\begin{array}{l}-471340.3 \\
-3352076\end{array}$ & $\begin{array}{l}19096.62 \\
1996\end{array}$ & 14.21 & 2.12 & 12.70 & 1.5 & $\begin{array}{l}0.80 \\
0.93\end{array}$ & $\begin{array}{l}41.3 \\
33.9\end{array}$ & $\begin{array}{l}0.35 \\
0.83\end{array}$ & $\begin{array}{l}0.23 \\
0.25\end{array}$ & $\begin{array}{r}82.2 \\
\end{array}$ & M0.5 & int & \\
\hline $\begin{array}{l}\text { J0213-3352 } \\
\text { J0213-7346 }\end{array}$ & LHS1356 & $\begin{array}{l}021239.48 \\
02125807\end{array}$ & $\begin{array}{l}-335207.6 \\
-734551.9\end{array}$ & $\begin{array}{l}1990.02 \\
1993.85\end{array}$ & 11.24 & 1.44 & 10.40 & 1.1 & $\begin{array}{l}0.93 \\
0.90\end{array}$ & 33.9 & 0.83 & 0.25 & 139.8 & M3.5 & I & \\
\hline $\begin{array}{l}\mathrm{J} 0213-7346^{7} \\
\mathrm{~J}(0218-5508\end{array}$ & & $\begin{array}{l}021258.07 \\
02181741\end{array}$ & $\begin{array}{l}-734551.9 \\
-5507346\end{array}$ & 1990.83 & 11.24 & 1.44 & $\begin{array}{l}10.40 \\
1087\end{array}$ & & 0.90 & $\begin{array}{l}41.5 \\
300\end{array}$ & & 0.25 & 104.3 & M1 & I & \\
\hline J0218-5508 & L1/4-34 & 021817.41 & $\begin{array}{l}-550734.6 \\
-651844.8\end{array}$ & 1989.73 & 14.93 & 1.69 & 10.87 & 1.3 & $\begin{array}{l}1.00 \\
0.86\end{array}$ & 30.0 & $\begin{array}{l}0.45 \\
0.51\end{array}$ & 0.20 & 69.7 & M2.5 & I & \\
\hline $\mathrm{J} 0220-6519^{8}$ & & 021955.74 & $\begin{array}{l}-651844.8 \\
-4546558\end{array}$ & 199073 & $\begin{array}{l}14.93 \\
14.05\end{array}$ & 2.0 & $\begin{array}{l}12.92 \\
1057\end{array}$ & 1.5 & 0.86 & 36.7 & 0.51 & 0.11 & 90.5 & M3.5 & I & \\
\hline J0224-4547 & & 02241 & -4546 & $\begin{array}{l}1990.73 \\
109166^{2}\end{array}$ & $\begin{array}{l}14.05 \\
1478\end{array}$ & $1 . \mathrm{f}^{\mathrm{r}} \mathrm{x}$ & 12. & 1.2 & 0.87 & $102.9 / 25.3$ & 0.33 & 0.19 & $185.3 / 45.6$ & M1 & $\mathrm{I} / \mathrm{int}$ & $75 / 25$ \\
\hline-8056 & & 02275 & -805 & 1991 & 14.78 & 1.8 & 12. & 1. & 0.94 & 46.2 & -0.17 & -0.34 & 83.2 & M3 & I & \\
\hline 25 & LHS5057 & 02322 & -2725 & 3 & 17.00 & 2.23 & 15.33 & 1.57 & 0.82 & $96.3 / 45.1$ & 0.38 & 0.23 & 200.8/94.1 & M4 & $\mathrm{I} / \mathrm{int}$ & $60 / 40$ \\
\hline $\begin{array}{l}\mathrm{J} 0233-2737 \\
\mathrm{~J} 2023-506\end{array}$ & $\begin{array}{l}\text { LHS1416 } \\
\text { L25-57 }\end{array}$ & 023241.86 & $\begin{array}{l}-273726.0 \\
-5305354\end{array}$ & 93 & $\begin{array}{l}14.06 \\
1078\end{array}$ & 2.60 & $\begin{array}{r}13.06 \\
070\end{array}$ & 1.36 & $\begin{array}{l}0.83 \\
0.90\end{array}$ & $\begin{array}{r}22.1 \\
96\end{array}$ & $\begin{array}{l}0.65 \\
0.5\end{array}$ & 0.37 & $\begin{array}{l}78.6 \\
101\end{array}$ & M3 & int & \\
\hline $\begin{array}{l}\mathrm{J} 0234-5306 \\
\mathrm{~J} 0241-6045\end{array}$ & $\begin{array}{l}\text { L2255-57 } \\
\text { L127-33 }\end{array}$ & $\begin{array}{l}023420.95 \\
024036.99\end{array}$ & $\begin{array}{l}-530535.4 \\
-60444.5\end{array}$ & $\begin{array}{l}1993.96 \\
199.78\end{array}$ & $\begin{array}{l}10.78 \\
13.85\end{array}$ & $\begin{array}{l}2.10 \\
1.50\end{array}$ & $\begin{array}{r}9.79 \\
12.34\end{array}$ & $\begin{array}{l}1.49 \\
1.13\end{array}$ & $\begin{array}{l}0.90 \\
0.91\end{array}$ & $\begin{array}{r}9.6 \\
116.7 / 30.4\end{array}$ & $\begin{array}{l}0.25 \\
0.19\end{array}$ & $\begin{array}{r}-0.34 \\
0.29\end{array}$ & $\begin{array}{r}19.1 \\
193.6 / 50.4\end{array}$ & $\begin{array}{l}\text { M3.5 } \\
\text { M0.5 }\end{array}$ & $\begin{array}{l}\mathrm{I} \\
\mathrm{I} / \mathrm{int} \\
\mathrm{s}\end{array}$ & $45 / 55$ \\
\hline J0244-5835 & & $\begin{array}{l}024035.99 \\
024356.21\end{array}$ & -5 & & 16. & 2.2 & 13. & 1. & 1. & 31.0 & 0.29 & 0. & 57.3 & M5 & I & \\
\hline $\mathrm{J} 02$ & LHS 14 & 02 & - & & 13 & 1. & 12 & 0. & 0. & $87.1 / 39.8$ & -0.51 & -0. & $244.3 / 111.7$ & M0 & int/II & $75 / 25$ \\
\hline $\mathrm{J} 02$ & LP993-119 & & & & 12 & 1. & 11 & 1. & 0. & 39.5 & 0.43 & 0.1 & 80.5 & M2.5 & I & \\
\hline & & & & & 15 & 2. & 13 & 1. & 0 & 35 & 0. & 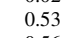 & 112.7 & M4 & I & \\
\hline $\mathrm{J} 02$ & LHS1448 & 0248 & & & 10 & 1. & & 1. & 0. & 4 & 0. & -0. & 223 & M0 & $\mathrm{I} / \mathrm{i}$ & $25 / 75$ \\
\hline J0250-2444 & LP830-44 & 024936.12 & -2444 & & 13 & 1. & 12 & 1.1 & 0.8 & $0.0 / 35.1$ & 0.3 & 0.6 & $219.8 / 59.9$ & M0.5 & $\mathrm{I} / \mathrm{int}$ & $20 / 80$ \\
\hline J0250-4941 & & 025004.24 & -494042.4 & 1992.81 & 15.68 & 2.29 & 14.17 & 1.39 & 0.87 & $108.1 / 34.3$ & 0.13 & 0.28 & 158.8/50.4 & M3 & $\mathrm{I} / \mathrm{int}$ & $80 / 20$ \\
\hline
\end{tabular}

${ }_{2}^{1}$ Distance is $13 \mathrm{pc}$ from the Yale parallax catalogue.

2 Distance is $17 \mathrm{pc}$ from the Yale parallax cotalogue, $23 \mathrm{pc}$ from the Hipparcos catalogue.

${ }_{4}^{3}$ Distance is $22 \mathrm{pc}$ from the Hipparcos catalogue.

${ }_{5}^{5}$ Distance is $13 \mathrm{pc}$ from the Yale parallax catalogue and the Hipparcos catalogue.

7 Distance is $18 \mathrm{pc}$ from the ARICNS.

8 Already identified by Scholz et al. (2002) at $34 \mathrm{pc}$. 


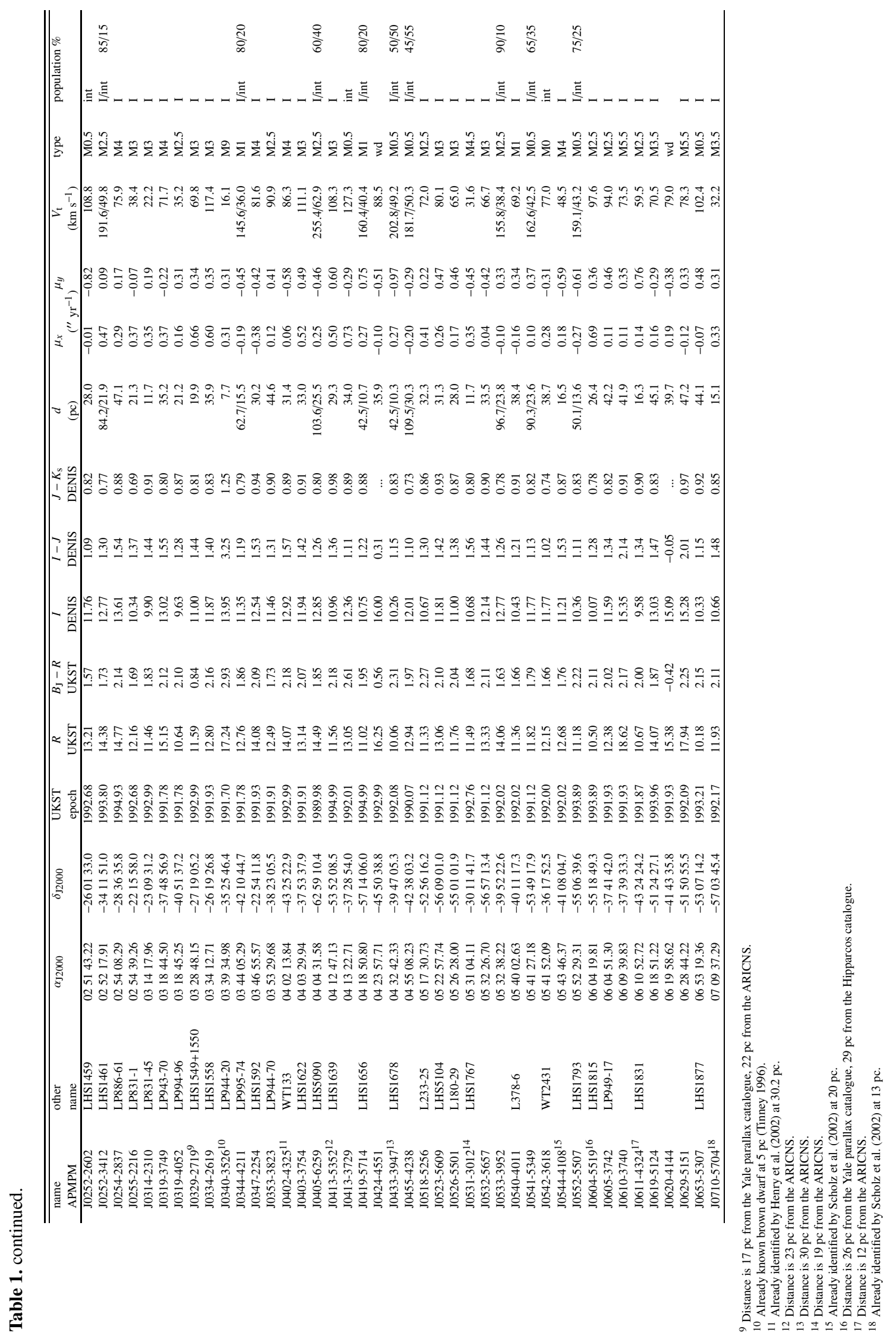




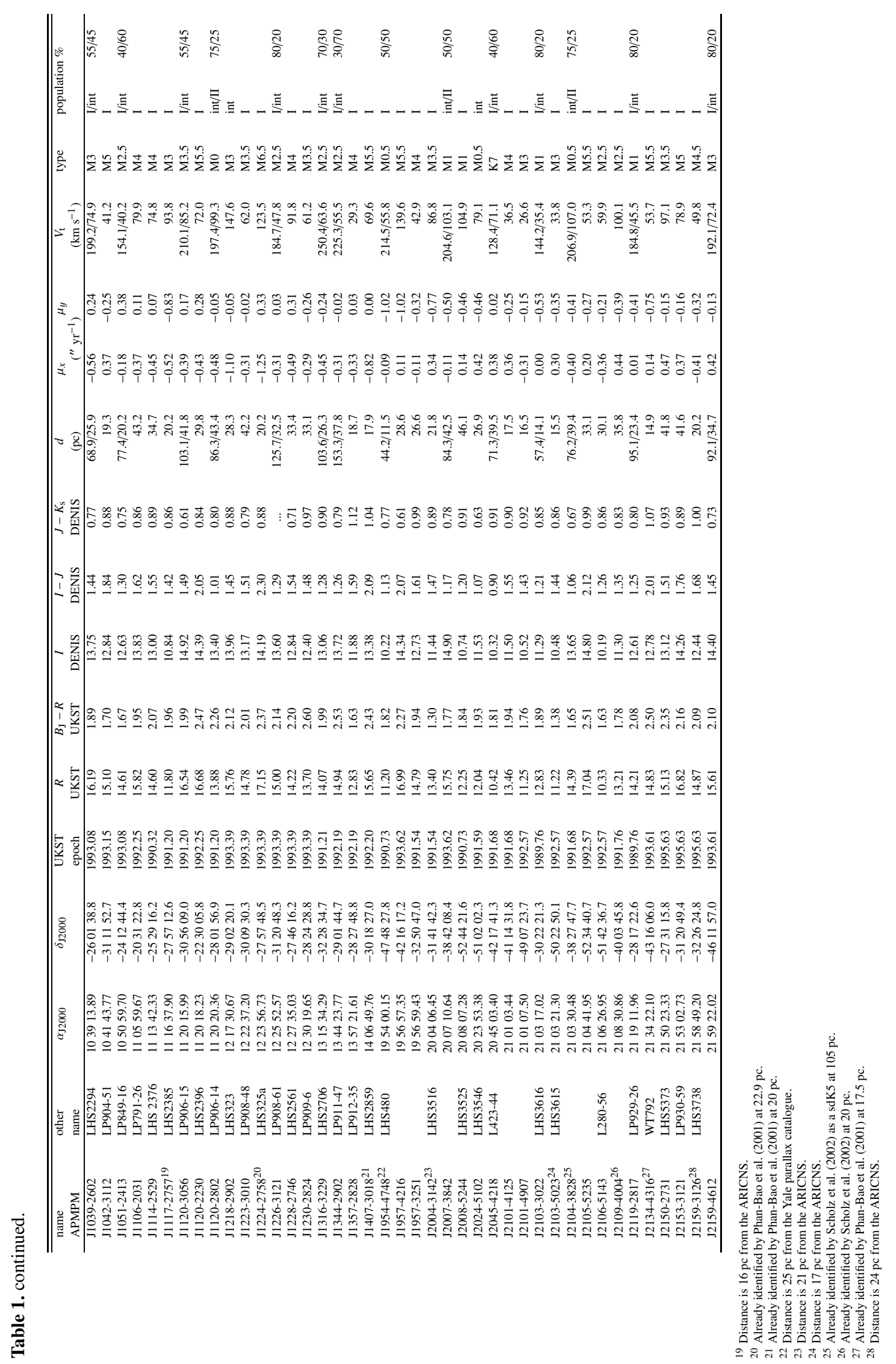




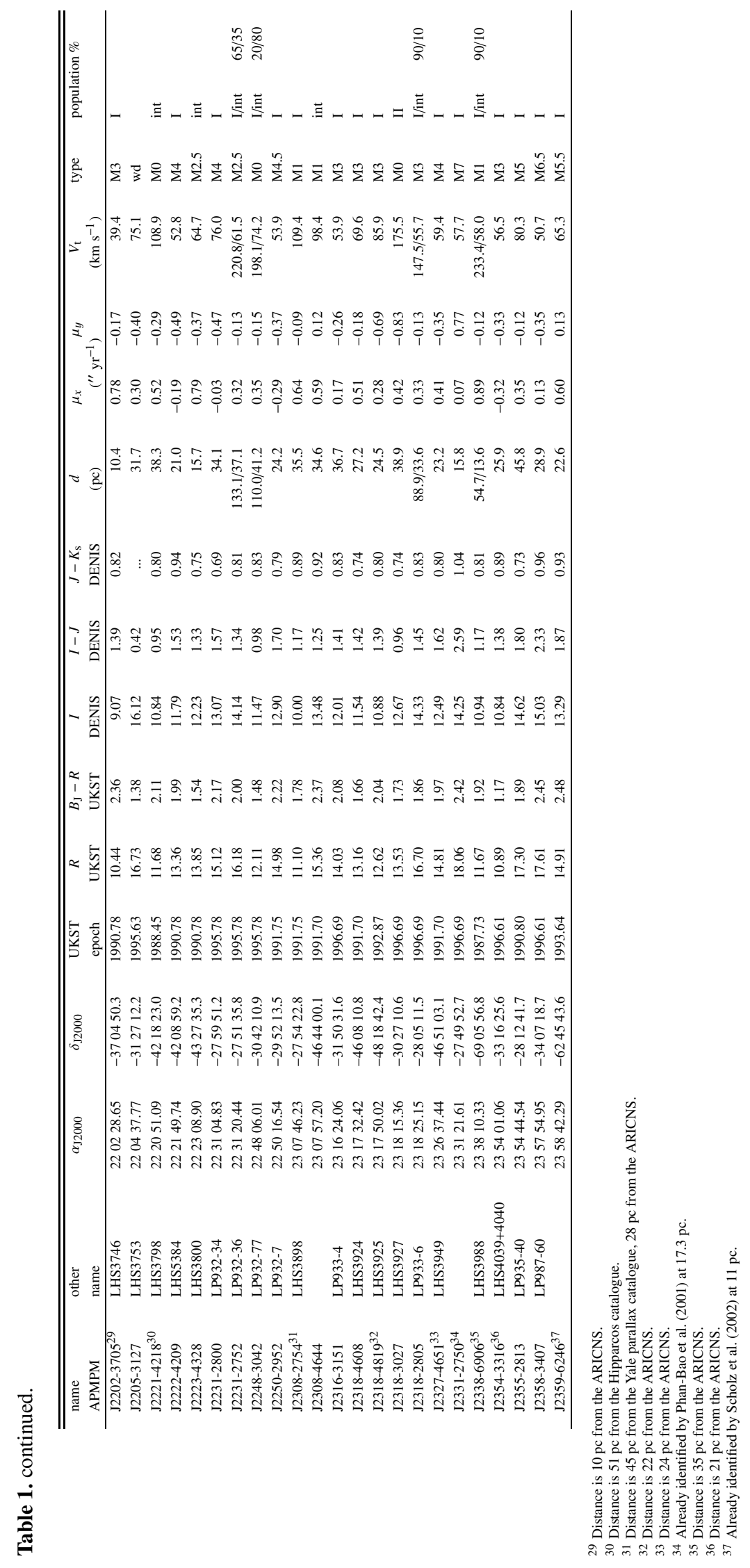




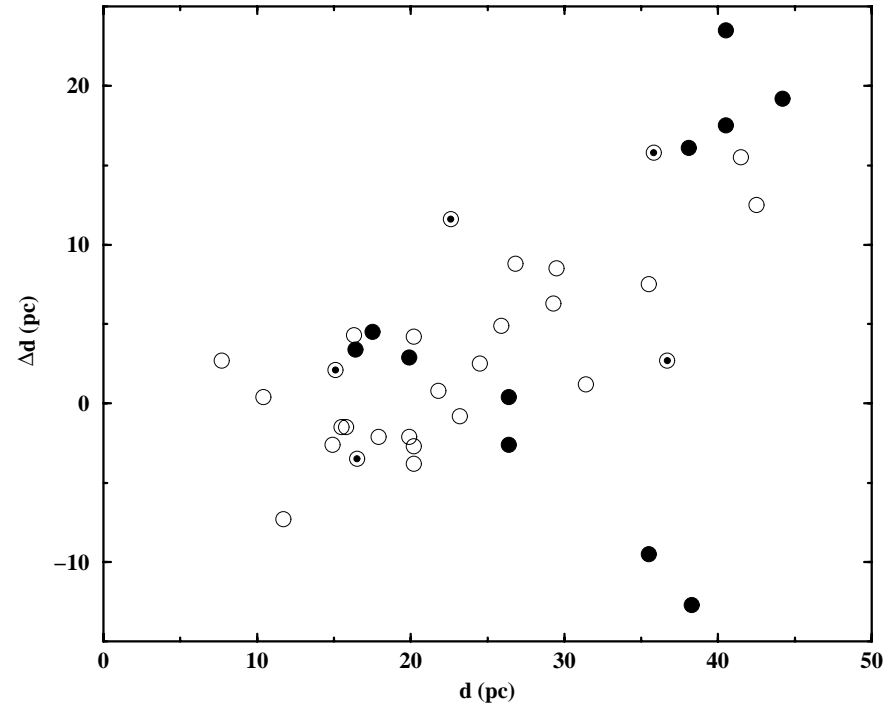

Fig. 3. Comparison between our estimated distances and determination by other authors, as indicated in Table $1 . \Delta d$ is the difference between both determinations. $d$ is our determination. Open circles: photometric determinations. Filled circles: trigonometric determinations. Circled dots: spectroscopic determinations.

It should be noted that the distances estimated in Table 1 have large uncertainties, particularly for stars with $I-J<$ 1.5 , for which the colour index is less sensitive to the luminosity (see Fig. 1). Moreover, Phan-Bao et al. (2001) plotted the colour-magnitude relation of M-stars with measured distances and noted an intrinsic scatter of \pm 1 mag. They also superimposed the Baraffe et al. (1998) theoretical track which does not fit perfectly the mean observed relation around $I-J=1.5$ where the $M_{I}$ magnitude is lower by 1 magnitude compared to the magnitude obtained with a polynomial fit of the observed data (Phan-Bao et al. 2001). For this reason, our distance determination for the 4 stars already identified by Phan-Bao et al. (2001) is smaller by a factor up to $15 \%$.

Figure 3 gives the difference between our estimated distance and other distances found in the litterature, as indicated in Table 1 footnotes, versus our determination. Open circles are used when the other determination is photometric, filled circles when it is trigonometric, circled dots when it is spectroscopic. Our determination is not biased compared to trigonometric parallaxes, giving confidence in our distances, while a systematic deviation appears at large distances between other photometric distances and ours, due to the different theoretical colourmagnitude relations used. The mean error is of the order of $5 \mathrm{pc}$ at distances closer than $25 \mathrm{pc}$ but grows to more than $10 \mathrm{pc}$ at larger distances.

\section{Conclusion}

Thanks to the deep and large surveys at optical and nearinfrared wavelengths, many low-luminosity nearby stars are identified. Most of the nearest stars turned out to have a large proper motion. In this paper, we present the identification of 107 stars closer than 50 pc among high proper motion stars cross-identified with DENIS. Their large proper motions exclude that they are giants. They are mainly M-stars, while 4 stars are white dwarfs. 31 stars among them have already measured distances. In addition, 40 stars may enter in the $50 \mathrm{pc}$ limit depending on which population they belong to. 6 stars among them have previously measured distances.

15 new stars are within the 25 pc limit of the CNS3 catalogue. 5 stars, LHS5045, L225-57, LP831-45, LHS1767, and WT792, are closer than 15 pc,with 1 object, L225-57, at 9.5 pc. In order to insure the spectral types and therefore distances, we plan to obtain low-resolution spectroscopy of the closest candidates.

Acknowledgements. The authors thank Guy Simon who provided photometric values in a strip not available in the PDAC database, the whole DENIS staff and all the DENIS observers who collected the data. The DENIS project is supported by the SCIENCE and the Human Capital and Mobility plans of the European Commission under grants CT920791 and CT940627 in France, by l'Institut National des Sciences de l'Univers, the Ministère de l'Éducation Nationale and the Centre National de la Recherche Scientifique (CNRS) in France, by the State of Baden-Würtemberg in Germany, by the DGICYT in Spain, by the Sterrewacht Leiden in Holland, by the Consiglio Nazionale delle Ricerche (CNR) in Italy, by the Fonds zur Förderung der wissenschaftlichen Forschung and Bundesministerium für Wissenschaft und Forschung in Austria, and by the ESO C \& EE grant A-04-046.

\section{References}

Baraffe, I., Chabrier, G., Allard, F., \& Hauschildt, P. H. 1998, A\&A, 337, 403

Bergeron, P., Wesemael, F., \& Beauchamp, A. 1995, PASP, 107, 1047

Dawson, P. C. 1986, ApJ, 311, 984

Delfosse, X. 1997, Ph.D. Thesis, Grenoble University

Delfosse, X., Tinney, C. G., Forveille, T., et al. 1997, A\&A, 327, L25

Delfosse, X., Tinney, C. G., Forveille, T., et al. 1999, A\&AS, 135, 41

Delfosse, X., Forveille, T., Martín, E. L., et al. 2001, A\&A, 366, L13

Epchtein, N., de Batz, B., Capoani, L., et al. 1997, The Messenger, 87, 27

Fan, X., Knapp, G. R., Strauss, M. A., et al. 2000, AJ, 119, 928

Gizis, J., \& Reid, I. 1997, PASP, 109, 849

Gizis, J. E., Monet, D. G., Reid, I. N., Kirkpatrick, J. D., Liebert, J., \& Williams, R. J. 2000, AJ, 120, 1085

Gliese, W., \& Jahreiß, H. 1991, in The Astronomical Data Center CD-ROM: Selected Astronomical Catalogs, vol. I; ed. L. E. Brotzmann, \& S. E. Gesser, NASA/Astronomical Data Center, Goddard Space Flight Center, Greenbelt, MD

Haywood, M., Robin, A. C., \& Crézé, M. 1997, A\&A, 320, 440

Henry, T. J., Ianna, P. A., Kirkpatrick, J. D., \& Jahreiss, H. 1997, AJ, 114,388

Henry, T. J., Walkowicz, L. M., Barto, T. C., \& Golimowski, D. A. 2002, AJ, preprint [astro-ph®112496]

Jahreiß, H., Scholz, R., Meusinger, H., \& Lehmann, I. 2001, A\&A, 370, 967

Kirkpatrick, J. D., Reid, I. N., Liebert, J., et al. 1999, AJ, 519, 802

Kirkpatrick, J. D., Reid, I. N., Liebert, J., et al. 2000, AJ, 120, 447

Leggett, S. K. 1992, ApJS, 82, 351

Lejeune, T., Cuisinier, F., \& Buser, R. 1997, A\&AS, 125, 229

Leonard, P. J. T., \& Tremaine, S. 1990, ApJ, 353, 486

Martín, E. L., Delfosse, X., Basri, G., et al. 1999, AJ, 118, 2466

Meillon, L., Crifo, F., Gomez, A. E., Udry, S., \& Mayor, M. 1997, ESA SP-402: Hipparcos - Venice '97, 402, 591 
Phan-Bao, N., Guibert, J., Crifo, F., et al. 2001, A\&A, 380, 590

Reid, I. N., Kirkpatrick, J. D., Gizis, J. E., et al. 2000, AJ, 119, 369

Reylé, C., \& Robin, A. C. 2001, A\&A, 373, 886

Robin, A. C., Reylé, C., \& Crézé, M. 2000, A\&A, 359, 103

Ruiz, M. T., Takamiya, M. Y., Mendez, R., Maza, J., \& Wishnjewsky, M. 1993, AJ, 106, 2575

Ruiz, M. T., Leggett, S. K., \& Allard, F. 1997, ApJ, 491, L107

Scholz, R.-D., Irwin, M., Schweitzer, A., \& Ibata, R. 1999, A\&A, 345, L55

Scholz, R.-D., Irwin, M., Ibata, R., Jahreiß, H., \& Malkov, O. Y. 2000, A\&A, 353, 958
Scholz, R.-D., Meusinger, H., \& Jahreiß, H. 2001, A\&A, 374, L12 Scholz, R.-D., Ibata, R., Irwin, M., Lehmann, I., Salvato, M., \& Schweitzer, A. 2002, MNRAS, 329, 109

Tinney, C. G. 1996, MNRAS, 281, 644

Wroblewski, H., \& Torres, C. 1989, A\&AS, 78, 231

Wroblewski, H., \& Torres, C. 1991, A\&AS, 91, 129

Wroblewski, H., \& Torres, C. 1994, A\&AS, 105, 179

Wroblewski, H., \& Torres, C. 1996, A\&AS, 115, 481

Wroblewski, H., \& Torres, C. 1997, A\&AS, 122, 447 